\title{
ACT em Grupo para Manejo de Ansiedade entre Universitários: Ensaio Clínico Randomizado
}

\author{
Raimundo Bittencourt de Almeida ${ }^{1}$ \\ ${ }^{1}$ Universidade Federal do Rio Grande, RS, Brasil. \\ Lauro Miranda Demenech ${ }^{1}$ \\ ${ }^{1}$ Universidade Federal do Rio Grande, RS, Brasil.
}

\author{
Paulo Gomes de Sousa-Filho ${ }^{1}$ \\ ${ }^{1}$ Universidade Federal do Rio Grande, RS, Brasil. \\ Lucas Neiva-Silva ${ }^{1}$ \\ ${ }^{1}$ Universidade Federal do Rio Grande, RS, Brasil.
}

Resumo: Estima-se que um a cada cinco estudantes universitários ao redor do mundo apresenta algum tipo de transtorno psicológico, dentre os quais os transtornos de ansiedade são os mais prevalentes. Este estudo consiste em um ensaio clínico randomizado com o objetivo de avaliar a eficácia e efetividade de um protocolo semiestruturado de psicoterapia em grupo baseado nos princípios da Terapia de Aceitação e Compromisso (ACT) para o tratamento de ansiedade entre estudantes universitários. Os participantes foram alocados aleatoriamente entre os grupos intervenção e controle. Os níveis de ansiedade pré e pós-intervenção foram avaliados por meio do instrumento General Anxiety Disorders-7 (GAD-7). A amostra final do estudo foi composta por 15 pessoas, dentre as quais 10 foram alocadas no grupo intervenção e 5, no grupo controle. Os resultados foram analisados de duas formas: a) por protocolo (Per-protocol analysis); e b) por intenção de tratar (Intention-to-treat Analysis). Este tratamento reduziu a sintomatologia ansiosa em $39,7 \%$ entre os pacientes que completaram o tratamento (por protocolo, $p=0,030$ ) e em $30,8 \%$ entre todos alocados para o grupo intervenção (i.e., por intenção de tratar, incluindo os dropouts, $p=0,035$ ), enquanto os controles não tiveram redução significativa no mesmo período. Portanto, recomenda-se a utilização deste protocolo dentro do contexto universitário como uma alternativa viável ao acompanhamento individual em situações de transtornos de ansiedade. Pesquisas futuras com amostras maiores podem contribuir na consolidação deste protocolo.

Palavras-chave: Terapia de Aceitação e Compromisso, Terapia de grupo, Transtornos de ansiedade, Estudantes universitários, Ensaio clínico randomizado.

\section{Group Therapy Based on ACT for Anxiety Management among University Students: Randomized Clinical Trial}

\begin{abstract}
One in every five university students around the world is estimated to have some type of psychological disorder, considering anxiety disorders as the most prevalent. This study consists in a randomized clinical trial aimed to evaluate efficacy and effectiveness of a semi-structured group psychotherapy protocol based on the principles of Acceptance and Commitment Therapy (ACT) for the treatment of anxiety among university students. Participants were randomly allocated in the intervention and control groups. Pre- and post-intervention anxiety levels were measured using the General Anxiety Disorders-7 (GAD-7) questionnaire. The study sample was composed of 15 people, 10 of whom were allocated to the intervention group and 5 to the control group. Results were analyzed in two ways: 1) Per protocol analysis; and 2) Intention-to-treat analysis. This treatment reduced anxiety symptoms by $39.7 \%$ among patients who completed treatment (Per protocol, $p=0.003$ ) and by $30.8 \%$ among all those allocated to the intervention group (i.e., by Intention-to-treat, including dropouts, $p=0.035$ ), whereas controls showed no
\end{abstract}


significant reduction in same period. Therefore, we recommend the use of this protocol within the university context as a viable alternative to individual counselling in situations of anxiety disorders. Future research with larger samples may contribute to consolidate this protocol.

Keywords: Acceptance and Commitment Therapy, Group therapy, Anxiety disorders, University students, Randomized controlled trial.

\title{
Terapia Grupal Basada en ACT para el Manejo de la Ansiedad entre Estudiantes Universitarios: Ensayo Clínico Aleatorizado
}

\begin{abstract}
Resumen: Se estima que uno de cada cinco estudiantes universitarios en todo el mundo tiene algún tipo de trastorno psicológico, entre los cuales los trastornos de ansiedad son los más frecuentes. Este estudio consiste en un ensayo clínico aleatorizado cuyo objetivo fue evaluar la eficacia y efectividad de un protocolo de psicoterapia grupal semiestructurada basado en los principios de la Terapia de Aceptación y Compromiso (ACT) para el tratamiento de la ansiedad en estudiantes universitarios. Se asignaron aleatoriamente a los participantes en los grupos de intervención y control. Los niveles de ansiedad antes y después de la intervención se midieron utilizando el instrumento General Anxiety Disorders-7 (GAD-7). La muestra final del estudio estuvo compuesta por 15 personas, de las cuales 10 fueron asignadas al grupo de intervención y 5 al grupo de control. Los resultados se analizaron de dos maneras: 1) por protocolo (Per protocol analysis); y 2) por intención de tratar (Intention-to-treat Analysis). Con este tratamiento los síntomas de ansiedad se redujeron en un $39,7 \%$ entre los pacientes que completaron el tratamiento (por protocolo, $p=0,030$ ) y en un $30,8 \%$ entre todos los asignados al grupo de intervención (es decir, por intención de tratar, incluidos los dropouts, $p=0,035$ ), mientras que los controles no tuvieron una reducción significativa en el mismo periodo. Por lo tanto, se recomienda utilizar este protocolo dentro del contexto universitario como una alternativa viable a lo asesoramiento individual en situaciones de trastornos de ansiedad. La investigación futura con muestras más grandes puede contribuir a la consolidación de esta modalidad.
\end{abstract}

Palabras clave: Terapia de Aceptación y Compromiso, Terapia grupal, Trastornos de ansiedad, Estudiantes universitarios, Ensayo clínico aleatorizado.

\section{Introdução}

Ansiedade e medo são respostas humanas naturais de extrema importância para a sobrevivência (Barlow \& Durand, 2015; Eifert \& Forsyth, 2014). A reação de medo é evocada na percepção de um perigo real ou imaginário, gerando uma ação defensiva que motiva e mobiliza o corpo para fugir da ameaça (Germer, Siegel, \& Fulton, 2015). A ansiedade pode ser entendida como um conjunto de reações fisiológicas e psicológicas, associadas a uma situação de medo excessivo (Barlow \& Durand, 2015). Trata-se de um sistema simbólico verbal de resposta cognitiva e fisiológica marcado por preocupações sobre eventos futuros potencialmente perigosos. Dessa forma, a ansiedade pode ser compreendida como um "estado de ameaça" (Eifert \& Forsyth,
2014). Ainda que a ansiedade possa desempenhar uma função importante em diversos âmbitos da vida (e.g., ambiente familiar, relacionamentos, trabalho e contexto acadêmico), o impulso de fuga de situações geradoras de medo, quando ocorre de forma rígida/ inflexível e/ou insensível às contingências contextuais, pode gerar sofrimento clínico (Eifert \& Forsyth, 2014). Em geral, os transtornos de ansiedade caracterizam-se por comportamentos rígidos e/ou ineficazes de esquiva diante de experiências avaliadas como indesejáveis e/ ou dolorosas (Twohig \& Levin, 2017).

O contexto acadêmico é pautado por exigências e demandas rígidas que ocupam um papel importante e complexo na vida dos estudantes universitários. Níveis elevados de estresse, competitividade, excesso de carga 
de trabalho, privação de sono, pressão e outros aspectos pessoais, curriculares, institucionais e afetivos constituem-se como barreiras para o desenvolvimento saudável (Pacheco et al., 2017). Dessa forma, as taxas de transtornos mentais entre universitários são superiores às encontradas na população geral (Auerbach et al., 2016; Ibrahim, Kelly, Adams, \& Glazebrook, 2013; World Health Organization [WHO], 2017). Um a cada cinco estudantes universitários ao redor do mundo tem algum tipo de transtorno psicológico, dentre os quais os transtornos de ansiedade são os mais prevalentes (Auerbach et al., 2016).

Em 2015, a proporção estimada da população global com algum transtorno de ansiedade foi de $3,6 \%$, o que representa aproximadamente 264 milhões de pessoas (WHO, 2017). De acordo com esse estudo, o Brasil é o país mais ansioso do mundo, com 9,3\% da população com algum transtorno de ansiedade. Dentro do contexto universitário, uma metanálise identificou que $37,75 \%$ dos graduandos brasileiros apresentam níveis clinicamente relevantes de ansiedade, que é mais prevalente entre estudantes do sexo feminino, nos anos iniciais e intermediários de seus cursos, com dificuldade de acesso a acompanhamento psicológico, insatisfeitos com seus cursos e com dificuldades de adaptação (Demenech, Oliveira, Neiva-Silva, \& Dumith, 2021).

A demanda por atendimento tem aumentado de forma intensa em todos os âmbitos da sociedade. Por sua vez, as universidades estão ficando sobrecarregadas com o aumento tanto na procura por acompanhamento psicológico por parte dos acadêmicos, como na severidade das situações de sofrimento (Gallagher, 2011; Pistorello, 2013; Twenge et al., 2010). A proposta tradicional de atendimento individual tem se mostrado eficaz (Roemer, Orsillo, \& SaltersPedneault, 2008; Twohig \& Levin, 2017). Entretanto, propostas de tratamento coletivas que contemplem maior número de pessoas podem se tornar importantes ferramentas para lidar com os desafios deste novo cenário. A literatura científica descreve abordagens terapêuticas que têm demonstrado resultados promissores no tratamento em grupo (Eilenberg, Fink, Jensen, Rief, \& Frostholm, 2015; Wolgensinger, 2015).

Existem diversas abordagens psicológicas que se propõem a lidar com os transtornos de ansiedade. A Terapia Cognitivo-Comportamental (TCC) tem sido apontada como aquela com o maior nível de evidência quanto a sua eficácia (Bandelow, Michaelis, \&
Wedekind, 2017). Contudo, a Terapia de Aceitação e Compromisso (ACT - Acceptance and Commitment Therapy) tem atraído a atenção de profissionais e pesquisadores desde sua formulação inicial (Hayes, Strosahl, \& Wilson, 1999, 2012) e estudos indicam tratar-se de uma abordagem possivelmente eficiente para o manejo de transtornos de ansiedade (Öst, 2014). A ACT estrutura-se a partir da filosofia pragmática do contextualismo funcional. Além disso, tem seus fundamentos em uma teoria orientada por um programa de pesquisa experimental sobre linguagem e cognição subjacente ao modelo ACT, chamada Teoria das Molduras Relacionais (RFT - Relational Frame Theory) (Hayes, Barnes-Holmes, \& Roche, 2002; Törneke, 2010). Dessa forma, a ACT tem uma visão sensível ao contexto e às funções dos eventos psicológicos, mais do que somente à forma como se apresentam (e.g., sintomas). Por meio de estratégias de mudanças contextuais e experienciais, busca-se construir um repertório amplo, flexível e efetivo para a mudança de função dos eventos aversivos (Hayes, Luoma, Bond, Masuda, \& Lillis, 2006).

A ACT propõe que o processo natural da mente humana conduz os indivíduos ao sofrimento, que é uma experiência humana presente cedo ou tarde em todos os domínios da vida. Tentativas de controle por meio da esquiva experiencial ocupam papel importante na maximização do sofrimento natural (Hayes et al., 2012). Apesar de a ansiedade estar relacionada a uma reação às situações possivelmente ameaçadoras, ao contrário do que acontecia com nossos antepassados (cuja ansiedade os mobilizava a resolverem e controlarem situações externas como a fome, frio, ameaça de predadores etc.), os desafios atuais do contexto acadêmico estão mais voltados a questões cognitivas e emocionais (e.g., lidar com a ansiedade de desempenho experienciada ao realizar uma prova, ao apresentar um seminário em público etc.).

Dessa forma, as estratégias de controle do ambiente externo que foram úteis no passado, por muitas vezes acabam ampliando o sofrimento psicológico (Eifert \& Forsyth, 2014). Ao tentar controlar pensamentos e emoções avaliados como desagradáveis, o indivíduo evoca, a partir da relação entre o não pensar ou não sentir, aquilo que justamente está buscando evitar (Hayes et al., 2012). Além disso, ao utilizar a esquiva experiencial para lidar com estímulos ansiogênicos/aversivos, a obtenção de alívio imediato funciona como um reforço negativo, o que 
aumenta a probabilidade de o comportamento de esquiva acontecer e deixar o sujeito preso em um círculo vicioso que restringe o repertório comportamental. Consequentemente, ocorre um distanciamento de áreas de vida importantes, amplificando o sofrimento (Hayes, Wilson, Gifford, Follette, \& Strosahl, 1996).

Para lidar com esse paradoxo, a ACT propõe a busca da mudança na relação dos indivíduos com a ansiedade e seus eventos dolorosos, ao invés de tentar controlá-los (Hayes et al., 1999, 2012). Para tal, busca-se desenvolver a flexibilidade psicológica, através de seis processos interdependentes (Hayes et al., 2006): a) Aceitação: busca a plena experiência com relação à condição humana, o contato aberto e consciente com o momento presente, sem defesas desnecessárias; b) Desfusão cognitiva: implica no distanciamento dos pensamentos, o que possibilita respostas baseadas em sua utilidade e não em rígidas verdades da mente; c) Eu como contexto: possibilita a percepção da experiência humana em constante mudança e considera o indivíduo como o palco onde os eventos acontecem; d) Clarificação de valores: busca a reflexão honesta acerca do que de fato importa na vida, os desejos mais profundos, as direções que verdadeiramente se deseja seguir; e) Ações comprometidas: busca o comprometimento com comportamentos alinhados aos valores de vida, ou seja, com o que os aproxima de uma vida rica e significativa; e f) Momento presente: diz respeito ao contato direto e contínuo com o "aqui-e-agora”, com menos julgamentos e mais descrições, tentando observar os eventos tal como são.

Ensaios clínicos têm demonstrado a eficácia de psicoterapia baseada nos princípios da ACT para o tratamento de diversos tipos de transtornos, incluindo os de ansiedade (England et al., 2012; Hayes-Skelton, Roemer, \& Orsillo, 2013; Kocovski, Fleming, Hawley, Huta, \& Antony, 2013; Roemer et al., 2008; Twohig \& Levin, 2017). No Brasil já existem diversos estudos e relatos sobre a aplicação da ACT para o tratamento de diversas condições, incluindo transtornos de ansiedade (Costa \& Soares, 2015; Fukahori, Silveira, \& Costa, 2005; J. Silva \& de-Farias, 2013; Sousa \& de-Farias, 2014; Vogel, Gordon, \& Lotufo Neto, 2017). Contudo, a utilização da ACT em modalidade grupal no país ainda é incipiente, com resultados de pesquisas para insônia (El Rafihi-Ferreira et al., 2020) e Transtorno de Humor Bipolar (Machado, Soares, \& Costa, 2019). Além disso, foi identificado apenas um estudo no Brasil relatando a aplicabilidade dos princípios da ACT em grupo para ansiedade (Chagas, Guilherme, \& Moriyama, 2013). Apesar de o referido trabalho indicar a possibilidade de resultados promissores, os autores concluem sobre a necessidade de serem conduzidos estudos controlados sobre a efetividade desse tipo de tratamento.

Considerando a situação atual do aumento do sofrimento psicológico de estudantes universitários, abordagens deste tipo podem servir como importantes ferramentas para os serviços de apoio ao estudante das universidades. Assim, o objetivo do presente estudo foi realizar um ensaio clínico randomizado, a partir do desenvolvimento de um protocolo semiestruturado de psicoterapia em grupo baseado nos princípios da ACT para o manejo de ansiedade clinicamente relevante em estudantes universitários, bem como apresentar resultados quantitativos sobre a eficácia e efetividade desta intervenção.

\section{Método}

\section{Delineamento}

Com o objetivo de avaliar a eficácia (capacidade de o tratamento reduzir sintomatologia ansiosa em contexto controlado) e efetividade (capacidade de o tratamento reduzir sintomatologia ansiosa em condições reais) da intervenção (Singal, Higgins, \& Waljee, 2014), este protocolo de tratamento foi submetido a um delineamento de estudo do tipo ensaio clínico randomizado (ECR). Trata-se de um projeto mais amplo desenvolvido pelo grupo de pesquisa, que incluiu três braços: a) controles; b) ACT em grupo; e c) TCC em grupo. Os resultados da intervenção em TCC não serão apresentados neste manuscrito, pois o objetivo desta publicação é apresentar aos profissionais da psicologia uma proposta de tratamento em grupo baseada na ACT, junto com os resultados preliminares sobre eficácia e efetividade desse tratamento. O protocolo desenvolvido utilizando a TCC, assim como os benefícios atingidos por essa intervenção, serão trabalhados de forma aprofundada em publicação futura.

\section{Recrutamento dos participantes}

Foi realizada ampla divulgação do projeto por meio de panfletos, cartazes, e-mails, página da internet e redes sociais da universidade e do grupo de pesquisa responsável. O material informativo incluía dados sobre os objetivos, formato da intervenção e condições para participação. A fim de popularizar e 
auxiliar na divulgação da proposta, este projeto foi nomeado como "Grupo para Redução de Ansiedade entre Universitários”, formando o acrônimo GRAU. O estudante interessado preenchia uma ficha de inscrição, disponibilizada na plataforma online Google Forms, na qual eram coletadas informações relevantes para avaliação da elegibilidade do candidato para participar do estudo.

\section{Critérios de elegibilidade e instrumentos}

Poderiam se inscrever para participar do estudo apenas universitários (de qualquer curso de graduação), de ambos os sexos, com idade igual ou superior a 18 anos, que estivessem regularmente matriculados na universidade. Como critério de inclusão, o candidato deveria apresentar níveis elevados de ansiedade. Os critérios de exclusão foram a presença de quadro depressivo clinicamente relevante e de risco alto de suicídio.

Para classificar os indivíduos em relação a sintomatologia ansiosa, depressiva e risco de suicídio, instrumentos traduzidos e validados para uso no Brasil foram inseridos na ficha de inscrição. Para mensurar a ansiedade foi utilizada a escala General Anxiety Disorders-7 (GAD-7) (Moreno et al., 2016; Spitzer, Kroenke, Williams, \& Lowe, 2006). Este instrumento lista sete problemas, sobre os quais os participantes respondem com que frequência têm se incomodado na última semana. As respostas, que podem ser "raramente", "alguns dias", "mais da metade dos dias" e "quase todos dias", são pontuadas de zero até três pontos. Estudantes com escore maior ou igual a 10 foram considerados como com ansiedade clinicamente relevante (sensibilidade $=89 \%$, especificidade $=82 \%$ ) (Moreno et al., 2016; M. Silva, Caicedo Roa, Martins, Silva, \& Galvao, 2018; Spitzer et al., 2006).

A sintomatologia depressiva foi medida por meio do Patient Health Questionnaire-9 (PHQ-9) (Kroenke, Spitzer, \&Williams, 2001; Santos et al., 2013). Esse instrumento é composto de nove perguntas sobre como a pessoa tem se sentido nas últimas duas semanas. As respostas possíveis para cada um dos questionamentos são "nenhum dia", "menos de uma semana", "uma semana ou mais" e "todos os dias", pontuando de 0 a 4 . Além disso, existe mais uma pergunta sobre o quanto estes sintomas estão dificultando o funcionamento do participante nas últimas duas semanas, que poderá responder "nenhuma dificuldade", "pouca dificuldade", "muita dificuldade" ou "extrema dificuldade". Estudantes com escores maiores ou iguais a nove nesse instrumento foram classificados como com presença de sintomatologia depressiva clinicamente relevante (sensibilidade $=77,5 \%$, especificidade $=86,7 \%$ ) (Santos et al., 2013).

Por fim, o risco de suicídio foi avaliado por meio do Mini International Neuropsychiatric Interview (MINI) (Amorim, 2000), adaptado para modelo autoaplicado. Neste instrumento, são feitas cinco perguntas, que se referem a se, durante o último mês: "Pensou que seria melhor estar morto(a) ou desejou estar morto(a)?", "quis fazer mal a si mesmo(a)?", "pensou em suicídio?", "pensou numa maneira de se suicidar?" e "tentou o suicídio?"; as perguntas valem 1, 2, 6, 10 e 10 pontos, respectivamente. Ainda, há uma última pergunta sobre tentativa de suicídio alguma vez na vida, que vale 4 pontos. Participantes com escore maior ou igual a nove são classificados como em risco alto de suicídio. Esse instrumento apresenta valores de validade globalmente satisfatórios em todas subescalas (sensibilidade $>0,70$, especificidade $>0,70$ ), apesar de o estudo de validação não apresentar esses dados específicos para as perguntas sobre suicídio (Amorim, 2000).

Estes critérios tiveram como objetivo selecionar os candidatos que se beneficiariam da intervenção proposta, além de objetivar, também, homogeneizar a demanda do grupo, que é uma característica importante para a adesão e eficácia das intervenções grupais (Pistorello, 2013). Dessa forma, tanto participantes com baixos níveis de ansiedade, como aqueles com significativa sintomatologia depressiva e/ou com risco alto de suicídio foram excluídos da pesquisa, sendo alocados, por motivos éticos, em outras modalidades de acompanhamento mais adequadas a suas necessidades. Portanto, como pode ser observado na Tabela 1, indivíduos com diferentes escores nas escalas avaliadas foram alocados da seguinte forma: a) aqueles com níveis baixos de ansiedade, depressão e risco de suicídio baixo ou ausente, foram direcionados para uma palestra de psicoeducação sobre ansiedade no ambiente universitário; b) candidatos com nível elevado de depressão e/ou risco alto de suicídio foram diretamente encaminhados para psicoterapia individual junto ao Centro de Atendimento Psicológico da universidade; e c) aqueles com ansiedade elevada e níveis de depressão e risco de suicídio baixos ou moderados, constituíram o grupo elegível para intervenção avaliada na presente pesquisa.

Todos os candidatos que preencheram os critérios para o GRAU ou para psicoterapia individual 
passaram por avaliação clínica por meio de entrevista individual. Nesta ocasião, foram explicados os objetivos da proposta de intervenção em grupo, bem como as possibilidades de alocação aleatória para os grupos intervenção ou controle.

\section{Tabela 1}

Critérios de inclusão e exclusão do projeto "Grupo para Redução de Ansiedade entre Universitários GRAU" e devidos encaminhamentos dos candidatos segundo os níveis de ansiedade, depressão e risco de suicídio. Rio Grande, RS, Brasil. 2019.

\begin{tabular}{cccc}
\hline & \multicolumn{3}{c}{ Instrumentos de avaliação } \\
\hline Desfecho & GAD-7 & PHQ-9 & $\begin{array}{c}\text { MINI- } \\
\text { Suicídio }\end{array}$ \\
\hline $\begin{array}{c}\text { Intervenção - } \\
\text { Psicoterapia em } \\
\text { grupo (GRAU) }\end{array}$ & $\geq 10$ & $<9$ & $\leq 9$ \\
$\begin{array}{c}\text { Encaminhamento - } \\
\text { Psicoeducação }\end{array}$ & $<10$ & $<9$ & $\leq 9$ \\
$\begin{array}{c}\text { Encaminhamento - } \\
\text { Psicoterapia } \\
\text { individual }\end{array}$ & Independente & $\geq 9$ & $>9$ \\
\hline
\end{tabular}

Nota. GAD-7 = General Anxiety Disorders-7; PHQ-9 = Patient Health Questionnaire-9; MINI-Suicídio = Mini International Neuropsychiatric Interview, seção suicídio; GRAU = Grupo para Redução de Ansiedade entre Universitários.

\section{Randomização}

Os participantes que preencheram os critérios de elegibilidade foram posteriormente alocados aleatoriamente em dois grupos: intervenção e controle. $\mathrm{Na}$ ficha de inscrição foram indicados dois horários diferentes para realização das atividades, para que os candidatos indicassem sua preferência, de acordo com suas disponibilidades. Nessa ocasião não era indicada nenhuma informação sobre qual abordagem teórica seria utilizada em cada um dos horários. Assim, a randomização foi feita de forma estratificada, ou seja, o grupo de candidatos foi dividido em dois, de acordo com a disponibilidade indicada pelos sujeitos, que foram posteriormente alocados de forma aleatória nos grupos intervenção e controle. Os nomes dos participantes foram substituídos por números e esse processo foi conduzido de forma cega por um dos membros da equipe de pesquisa que não participou do presente estudo. Aqueles alocados no grupo intervenção foram contatados para que recebessem as informações sobre o início do tratamento. O grupo controle consistiu em uma lista de espera.
Contudo, como uma medida ética, todos os indivíduos alocados no grupo controle foram considerados automaticamente incluídos no grupo intervenção oferecido no semestre subsequente.

\section{Avaliações pré e pós-intervenção}

Para que fosse possível avaliar a eficácia e efetividade do tratamento, foram realizadas duas avaliações, uma pré e outra pós-intervenção. A avaliação dos níveis de ansiedade pré-intervenção consistiu nos escores do GAD-7 obtidos pela triagem inicial. Após o período da intervenção, os participantes dos grupos intervenção e controle responderam novamente ao mesmo questionário, disponibilizado na mesma plataforma.

\section{Intervenção}

A intervenção consistiu em um grupo terapêutico com 12 encontros semiestruturados a partir dos pressupostos da ACT, com duração de aproximadamente $1 \mathrm{~h} 30 \mathrm{~min}$ e periodicidade semanal. A equipe foi formada por três terapeutas, todos previamente treinados para a execução do trabalho: um terapeuta principal, responsável pela condução do grupo (psicólogo em formação em ACT); um coterapeuta, responsável por auxiliar a condução do grupo (graduando em psicologia), bem como por estar atento às nuances que pudessem passar desapercebidas pelo terapeuta principal; e um observador (graduando em psicologia), responsável por fazer todos os registros das sessões, bem como contribuir quando necessário (Neufeld, 2017). O grupo ainda contava com um supervisor técnico (psicólogo com mais de cinco anos de prática clínica) e dois supervisores acadêmicos (ambos com mais de quinze anos de prática clínica, incluindo formação em ACT). O projeto contava com rotinas estabelecidas de duas horas semanais de supervisão em grupo para discussão sobre as sessões ocorridas e planejamento dos próximos encontros, bem como supervisões individuais quando necessário.

Todos as sessões iniciaram com uma verificação de desconforto percebido que consistia em escolher um número de um até cinco (sendo $1=$ nenhum desconforto e 5 = extremamente desconfortável), referentes ao quão desconfortáveis sentiam-se naquele dia. O objetivo era promover autopercepção com relação ao desconforto, assim como identificar dificuldades em se expor e observar a evolução no decorrer do tratamento. Após esta verificação, era perguntado ao participante 
sobre como sua semana havia transcorrido. A partir de seus relatos, eram feitas considerações por parte dos terapeutas. A descrição das 12 sessões é apresentada na Tabela 2. As principais obras que ofereceram subsídio teórico e técnico para a elaboração deste protocolo foram: a) La terapia de aceptación y compromiso para trastornos de ansiedad (Eifert \& Forsyth, 2014); b) Acceptance and Commitment Therapy: the process and practice of mindful change (Hayes et al., 2012); c) Liberte-se: Evitando as armadilhas da procura da felicidade (Harris, 2011); e d) Get out of your mind \& into your life (Hayes \& Smith, 2005).

\section{Tabela 2}

Descrição dos objetivos e procedimentos adotados nas sessões do Grupo para Redução de Ansiedade entre Universitários (GRAU). Rio Grande, RS, Brasil, 2019.

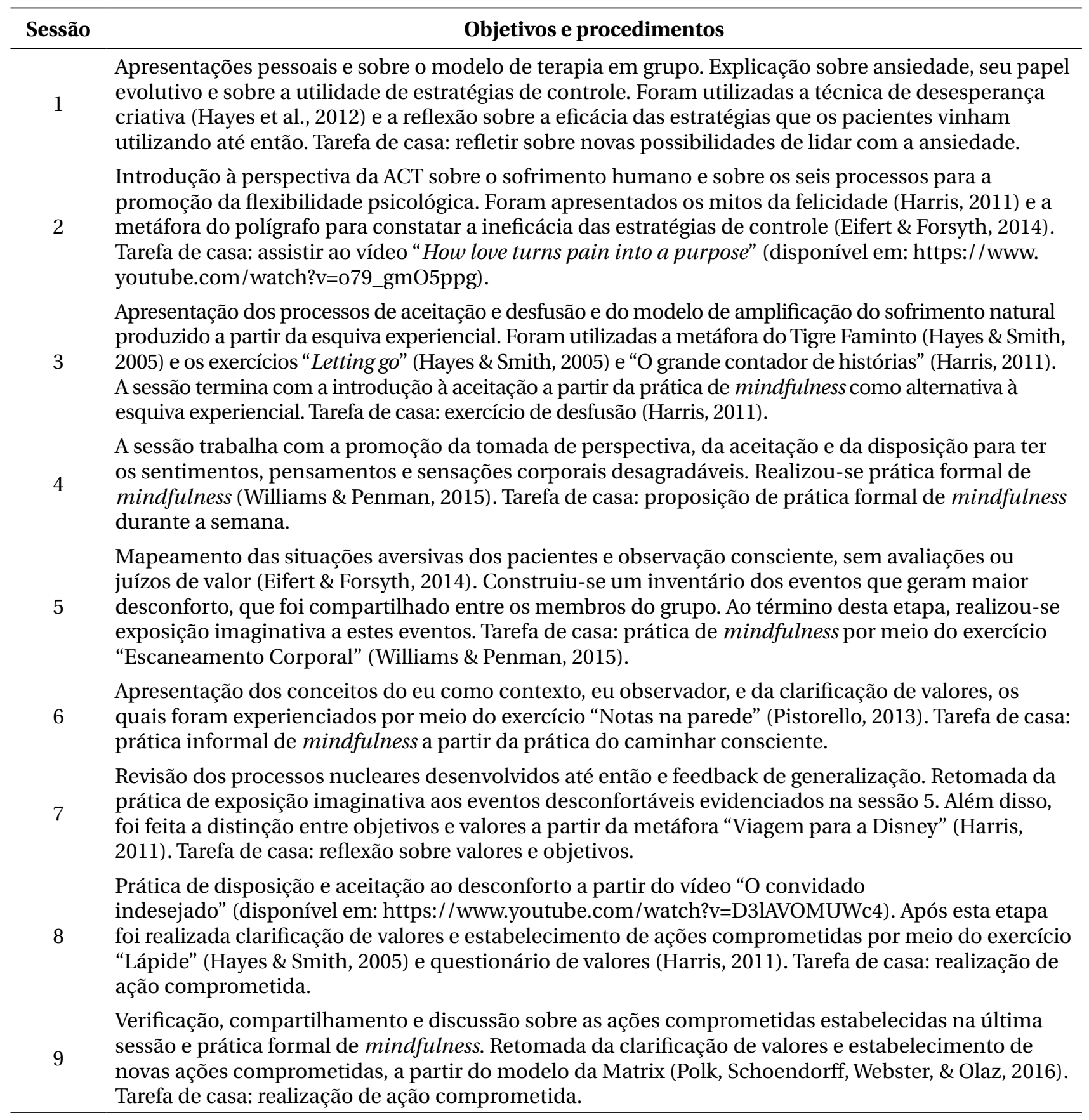


...continuação

\begin{tabular}{cl}
\hline Sessão & \multicolumn{1}{c}{ Objetivos e procedimentos } \\
\hline 10 & $\begin{array}{l}\text { Verificação, compartilhamento e discussão sobre as ações comprometidas estabelecidas na última } \\
\text { sessão e prática formal de mindfulness. Revisão das habilidades de desfusão, conexão e disposição. Foi } \\
\text { feito o estabelecimento de novas ações comprometidas e, posteriormente, foi realizado feedback de } \\
\text { generalização. Tarefa de casa: realização de ação comprometida estabelecida na sessão durante a semana. }\end{array}$ \\
& $\begin{array}{l}\text { Verificação, compartilhamento e discussão sobre as ações comprometidas estabelecidas na última } \\
\text { sessão e prática formal de mindfulness. Discussão sobre valores e esquiva experiencial, que foi } \\
\text { trabalhada a partir do exercício de autocompaixão (Neff, 2017), com o objetivo de promover }\end{array}$ \\
& $\begin{array}{l}\text { aceitação. Foram estabelecidas novas ações comprometidas. Tarefa de casa: realização de ação } \\
\text { comprometida estabelecida na sessão durante a semana. }\end{array}$ \\
& $\begin{array}{l}\text { Verificação, compartilhamento e discussão sobre as ações comprometidas estabelecidas na última } \\
\text { sessão e prática formal de mindfulness. Retomada dos principais conceitos trabalhados durante as } \\
\text { sessões do grupo e construção de ações comprometidas e metas de vida - de curto, médio e longo } \\
\text { prazo - alinhadas aos valores dos participantes (Harris, 2011). Ao fim, foi disponibilizada uma }\end{array}$ \\
cartilha com instruções para momentos de crise adaptada de Harris (2011).
\end{tabular}

Nota. Todas as sessões iniciavam com a partilha das reflexões e experiências evocadas a partir da tarefa de casa.

\section{Faltas e abandono}

Uma questão importante sobre a terapia em grupo é o abandono do tratamento. Por se tratar de um grupo para a redução de ansiedade, pressupõe-se que a participação nas sessões se constitui também como um estímulo ansiogênico. Por isso, é possível que os pacientes desistam do tratamento como uma forma de esquiva experiencial. Quando o paciente não comparecia à sessão, a equipe fazia contato para marcar um encontro de atualização sobre o que foi trabalhado durante a sessão correspondente. Em geral, esses encontros ocorriam 30 minutos antes da sessão da próxima semana, com o objetivo de treinar habilidades importantes para a manutenção do tratamento em grupo, bem como manter o paciente vinculado. Pacientes que faltassem a duas sessões consecutivas ou a quatro sessões no total, sem justificativa para a ausência, foram considerados como abandonos.

\section{Análises estatísticas}

As análises estatísticas foram conduzidas no software STATA IC 13.1. Primeiramente foram conduzidas análises univariadas para descrever a amostra. Posteriormente, foram realizadas análises bivariadas a fim de identificar possíveis diferenças significativas das características socioeconômicas e demográficas, bem como dos níveis iniciais de ansiedade, entre os grupos intervenção e controle. Para isso, foram utilizados o teste exato de Fisher (para diferenças de proporções) e o teste U de Mann-Whitney (para diferenças entre médias). As diferenças entre os escores no instrumento GAD-7 pré e pós-intervenção nos grupos intervenção e controle foram analisadas de duas formas. Para avaliar a eficácia do tratamento, foi utilizada a Análise por Protocolo (Per-protocol analysis). Para avaliar a efetividade do programa, foi utilizada a Análise por intenção de tratar (Intention-to-treat analysis). Em ambas as situações, a intervenção foi comparada ao grupo controle. Considerando o tamanho amostral, estratégias não-paramétricas foram adotadas e as diferenças nos escores de ansiedade pré e pós-intervenção foram avaliadas através do Wilcoxon signed rank test (Kirkwood \& Sterne, 2006). As estimativas foram calculadas considerando o nível de significância de $5 \%$ para testes bicaudais.

\section{Aspectos éticos}

Esta pesquisa foi aprovada pelo Comitê de Ética em Pesquisa na Área da Saúde (Cepas) da Universidade Federal do Rio Grande (FURG), registrado sob o parecer número 109/2019. O protocolo de tratamento proposto neste estudo foi registrado no Registro Brasileiro de Ensaios Clínicos (registro: RBR-6wnw9t).

\section{Resultados}

Após divulgação da abertura de vagas foram recebidas um total de 51 inscrições. Dezesseis indivíduos foram excluídos pois não tinham disponibilidade de participar do grupo nos horários pré-estabelecidos ou porque desistiram de suas candidaturas antes do 
início da intervenção. Dessa forma, 35 participantes passaram pela triagem, composta por formulário online e entrevista clínica. Dentre os triados, cinco foram encaminhados para psicoterapia individual, pois eles apresentaram risco de suicídio elevado. Nenhum dos candidatos foi alocado no grupo de psicoeducação. Assim, 30 candidatos foram aleatoriamente alocados em três grupos distintos: 10 para o grupo intervenção em ACT, 8 para o grupo controle e 12 para o grupo intervenção em TCC (não incluído neste estudo). Dentre os 10 pacientes da intervenção em ACT, 8 completaram todo o protocolo do tratamento e 2 desistiram (Figura 1). Dessa forma, a taxa de abandono do tratamento foi de $20 \%$. A frequência dos participantes que concluíram o tratamento variou entre $67 \%$ e $100 \%$. Dentre os 8 participantes do grupo controle, foi possível obter a avaliação pós-intervenção de 5 pacientes.

A amostra final do estudo foi composta por 15 pessoas ( 10 do grupo intervenção e 5 do grupo controle), de cursos diferentes (exceto por dois sujeitos que estavam na mesma graduação, mas que não eram colegas), cujas características são descritas na Tabela 3. A média do escore do GAD-7 pré-intervenção foi de 16,47 em toda a amostra. Os participantes foram majoritariamente do sexo feminino $(86,7 \%)$, com idades entre 18 e 26 anos $(93,2 \%)$ e com renda familiar média de 1,72 salários mínimos. Não foram identificadas diferenças estatisticamente significativas em relação a sexo, idade, migração acadêmica, renda, nem de nível de ansiedade entres os grupos intervenção e controle (Tabela 3).

Tabela 3

Escore médio de ansiedade pré-intervenção e descrição dos grupos intervenção e controle de acordo com variáveis socioeconômicas e demográficas. Rio Grande, RS, Brasil. 2019.

\begin{tabular}{lcccc}
\hline \multicolumn{1}{c}{ Variável } & Total & Intervenção & Controle & $p$ \\
\hline Sexo (N=15) & & & & $0,524^{\mathrm{a}}$ \\
Feminino & $13(86,7 \%)$ & $8(80,0 \%)$ & $5(100,0 \%)$ & \\
Masculino & $2(13,3 \%)$ & $2(20,0 \%)$ & $0(0,0 \%)$ & \\
Idade (N=15) & & & & $0,364^{\mathrm{a}}$ \\
18 a 22 anos & $7(46,6 \%)$ & $3(30,0 \%)$ & $4(80,0 \%)$ & \\
23 a 26 anos & $7(46,6 \%)$ & $6(60,0 \%)$ & $1(20,0 \%)$ & \\
27 anos ou mais & $1(6,8 \%)$ & $1(10,0 \%)$ & $0(0,0 \%)$ & $0,887^{\mathrm{c}}$ \\
Renda familiar média em salários mínimos $(\mathrm{N}=14)^{\mathrm{b}}$ & 1,72 & 1,55 & 2,15 & $0,122^{\mathrm{c}}$ \\
Escore médio Ansiedade pré intervenção $(\mathrm{N}=15)$ & 16,47 & 15,60 & 18,20 & \\
\hline
\end{tabular}

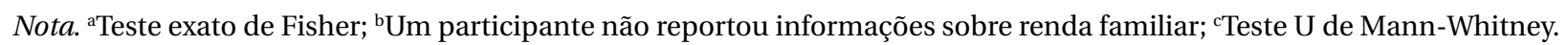

Os escores de ansiedade dos indivíduos alocados na intervenção e no controle antes e depois da intervenção podem ser observados na Figura 2. Quando os dados são observados a partir da Análise por Protocolo, identificou-se que o escore médio de ansiedade no grupo intervenção foi de 15,125, havendo uma redução significativa para 9,125 $(p=0,030)$. Isto representa uma redução de $39,7 \%$ (IC95\% 15,2\%: 64,1\%) no nível médio de ansiedade entre os tempos pré e pós-intervenção. Quando inseridos os pacientes que abandonaram o tratamento na análise, isto é, fazendo a Análise por Intenção de Tratar, observa-se que o escore médio de ansiedade no início do projeto entre os indivíduos do grupo intervenção foi de 15,6, tendo reduzido para 10,8 ( $p=0,035)$ no momento pós-intervenção. Portanto, a redução média do nível de ansiedade foi de $30,8 \%$ (IC95\% 4,4\%: 57,1\%). Os escores pré e pós-intervenção entre os controles foram 18,2 e 13,8, respectivamente. Isto corresponde a uma redução de $24,2 \%$ (IC95\% 11,4\%: 59,8\%), em que não se observou diferença estatisticamente significativa $(p=0,130)$. 


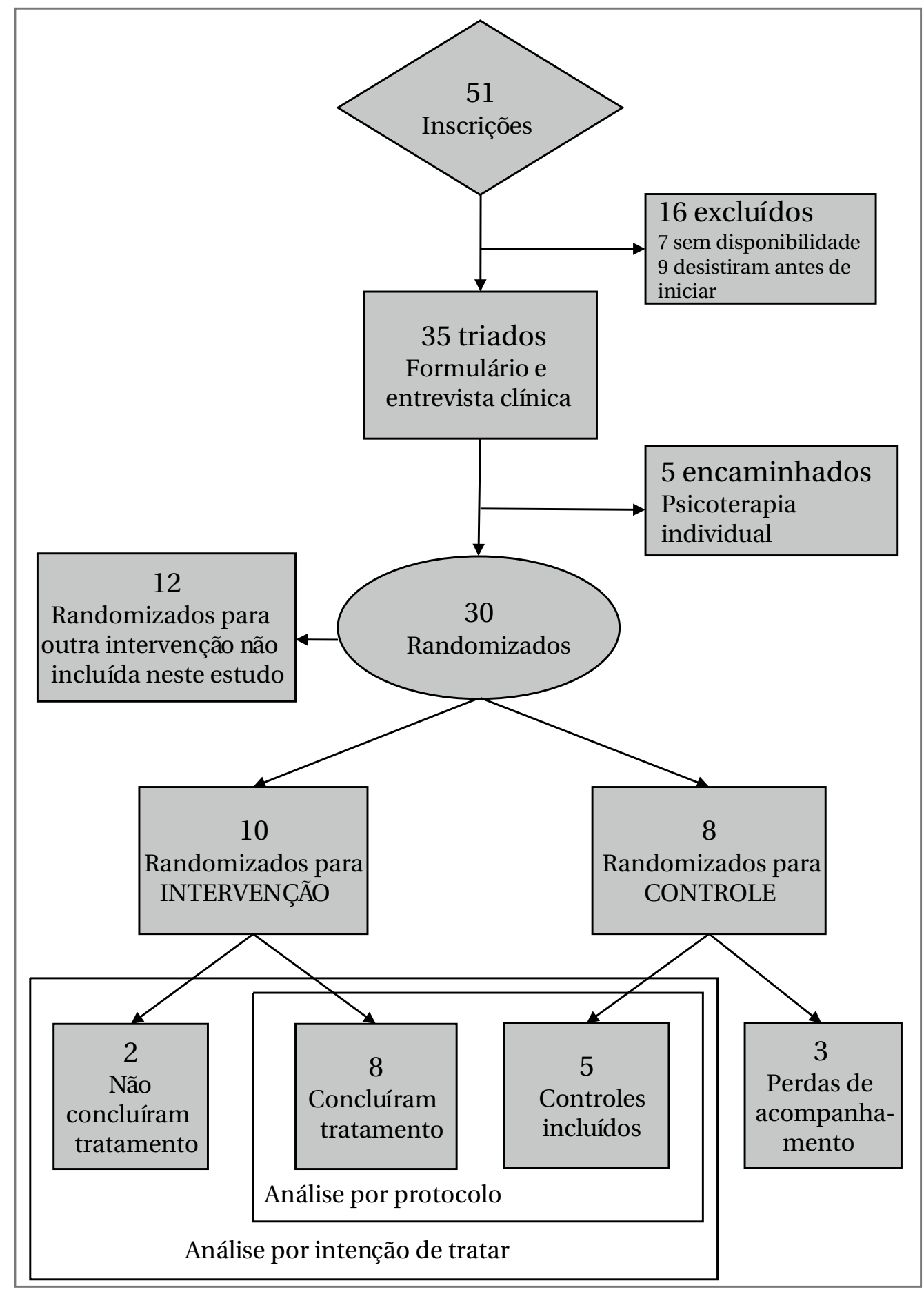

Figura 1

Fluxograma da alocação dos candidatos ao GRAU. Rio Grande, RS, Brasil, 2019.

\section{Discussão}

No presente estudo, a partir da comparação dos escores pré e pós-intervenção, observou-se redução significativa dos níveis de ansiedade entre os universitários do grupo intervenção, enquanto não se observou redução significativa nos níveis de ansiedade no grupo controle, no mesmo período. Este resultado sugere que a referida intervenção em grupo baseada na ACT tende a ser eficaz e efetiva. Pesquisas sobre ACT em grupo ainda são incipientes no Brasil. Mesmo assim, essa investigação vai ao encontro dos resultados identificados por outros grupos de pesquisa brasileiros. 
Por exemplo, protocolos de tratamento em grupo a partir dos pressupostos da ACT mostraram-se eficazes para o manejo de transtorno de humor bipolar (Machado et al., 2019) e de insônia (El Rafihi-Ferreira et al., 2020). Ainda, em um estudo sobre ACT em grupo para ansiedade, por meio de um relato de experiência, os autores concluíram que a proposta parecia exercer efeito positivo no manejo de ansiedade, mas que estudos futuros com experimentos controlados poderiam confirmar essa hipótese (Chagas et al., 2013). Dessa forma, a presente investigação se soma ao corpo de evidências que dá sustentação para tal afirmação.

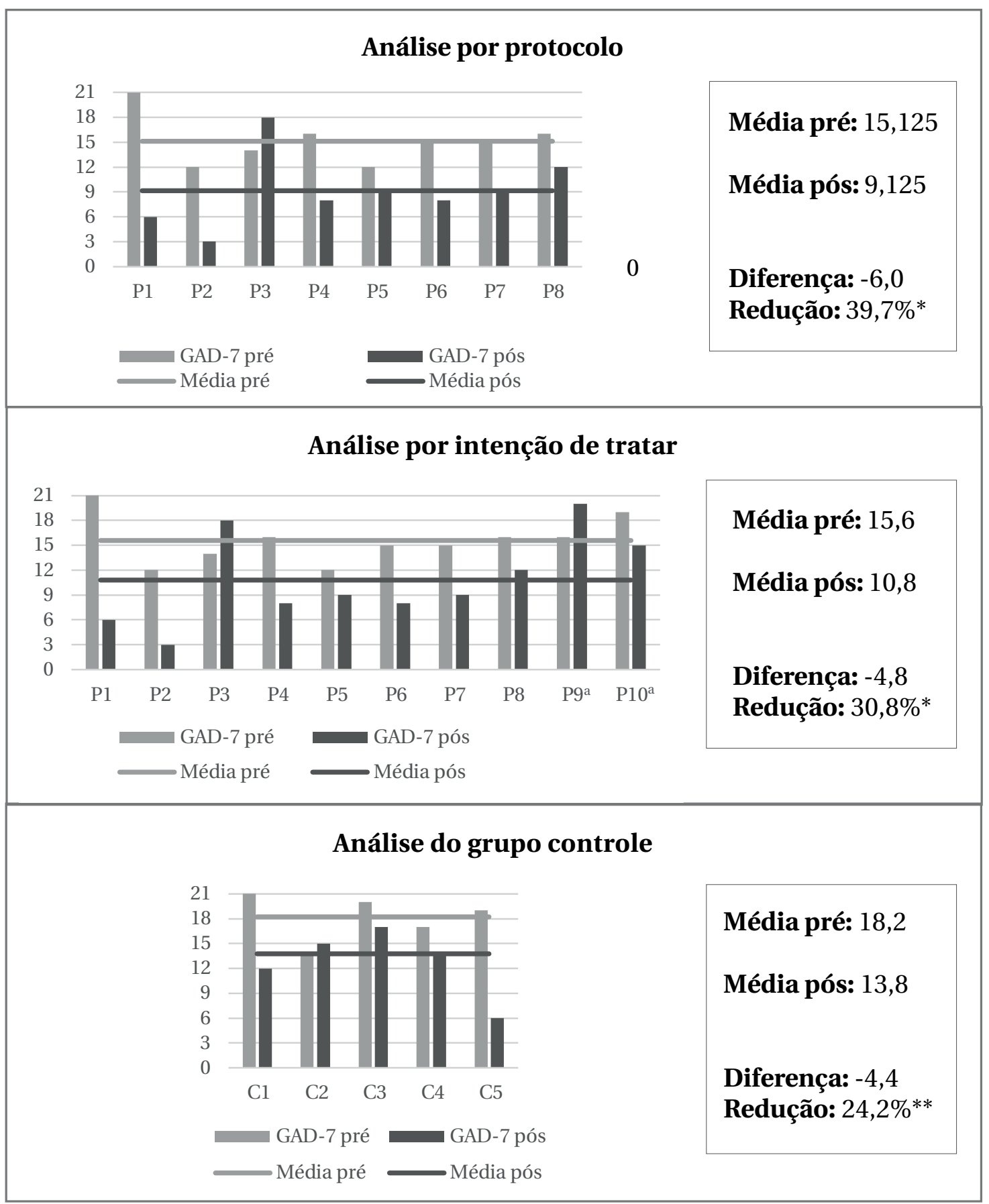

Figura 2

Gráficos das análises dos níveis de ansiedade pré e pós-intervenção nos grupos intervenção e controle. Rio Grande, RS, Brasil, 2019.

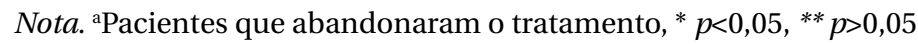


Por outro lado, existe um número maior de publicações sobre a utilidade da ACT em grupo para o manejo de ansiedade oriundas de estudos internacionais. Em uma revisão sistemática recente, foram identificados 11 estudos sobre esse assunto e, assim como na presente investigação, os resultados de todos apontam para a eficácia dessa modalidade de tratamento (Coto-Lesmes, Fernández-Rodríguez, \& GonzálezFernández, 2020). Os resultados desta intervenção também vão ao encontro (e se somam ao corpo de evidências) de uma atualização sobre a eficácia da ACT para diversas condições, que indicou que essa modalidade é possivelmente terapêutica para tratamento de ansiedade (Öst, 2014).

Dentre os artigos incluídos na revisão de CotoLesmes et al. (2020), apenas um foi conduzido com estudantes universitários, tratando-se de um relatório preliminar baseado em um estudo de caso de quatro pacientes atendidos por meio da ACT em grupo em uma universidade dos Estados Unidos. De acordo com a autora, as sessões do grupo auxiliaram os pacientes a reduzirem a esquiva experiencial, diminuindo os níveis de ansiedade como um produto deste processo (Daltry, 2015). É plausível que este também seja o fenômeno subjacente na redução de ansiedade observada nos participantes da intervenção aqui apresentada. Dessa forma, o presente estudo contribui para a compreensão da eficácia e efetividade da utilização de ACT em grupo para manejo de ansiedade entre universitários. Assim como na presente investigação, dois estudos utilizaram protocolos estruturados em 12 sessões (Jacobs, Luci, \& Hagemann, 2018; Malmir, Jafari, Ramezanalzadeh, \& Heydari, 2017) e dois mensuraram níveis de ansiedade por meio do instrumento GAD-7 (Jacobs et al., 2018; Perry, Gardener, Oliver, Taş, \& Özenç, 2019), o que indica a concordância e aplicabilidade dos métodos aqui utilizados.

Dentre os oito pacientes que completaram o tratamento, um (P3) não apresentou redução de sintomas. Isso pode ter ocorrido por três motivos. Primeiro, este paciente estava no período de conclusão de seu curso de graduação no momento em que respondeu ao questionário pós-intervenção. Assim, é possível que a ansiedade pontual do momento de resposta do questionário seja de fato elevada, mas que não necessariamente represente uma piora no quadro geral do paciente, mas sim uma resposta natural ao contexto.
Segundo, P3 apresentou desde o início da intervenção uma divergência entre os níveis de ansiedade reportados por meio do GAD-7 e a avaliação clínica dos terapeutas. Portanto, é possível que o aumento da ansiedade seja um reflexo da tomada de perspectiva e das ações comprometidas com seus valores (Hayes et al., 2012). Dessa forma, P3 pode ter aprendido a identificar com maior clareza seus sentimentos e pensamentos e se engajado em ações relacionadas a domínios importantes de sua vida, o que por definição pode gerar ansiedade (Lucena-Santos, Pinto-Gouveia, \& Oliveira, 2015). Por fim, é possível que, de fato, a intervenção não tenha produzido redução na sintomatologia ansiosa deste paciente.

Estar em grupo por si só pode constituir-se como uma exposição ao estímulo aversivo (e.g., falar em público), eliciando diversos eventos privados nos participantes. As sessões do grupo se mostraram como estímulos ansiogênicos, pois geraram desconforto em todos os participantes. Uma das preocupações foi de desenvolver estratégias que rompessem com o padrão de esquiva, que é mantido por meio do reforço negativo (Zamignani \& Banaco, 2005), ou seja, a esquiva tende a gerar um alívio instantâneo pela "retirada" de um estímulo aversivo. O êxito em experimentar de forma disposta a ansiedade provocada por esta proposta, por meio da emissão de respostas não evitativas e alinhadas com os valores dos participantes, mostrou-se nuclear na quebra do ciclo de retroalimentação da ansiedade e, consequentemente, no aumento do bem-estar dos pacientes.

É possível que a estratégia de marcar encontros "de reposição" da sessão perdida com os indivíduos tenha auxiliado na adesão dos participantes. Acredita-se que o mecanismo que explica este fenômeno é o desenvolvimento de habilidades específicas e necessárias (como a conexão com o momento presente, prática de desfusão etc.), considerando a experiência de cada um, com relação aos processos abordados nas sessões. Mais especificamente, a oportunidade de praticar alguns dos seis processos além do momento do grupo pode ter permitido que pacientes com diferentes históricos comportamentais tenham se comprometido com o grupo de maneira semelhante.

Em relação ao protocolo apresentado, destaca-se que sua construção se baseou nos seis processos centrais da ACT cujo objetivo foi promover um espaço 
para a promoção de flexibilidade psicológica. Apesar de o sofrimento ser uma consequência natural de estar vivo, as pessoas sofrem de forma desnecessária quando seus níveis de inflexibilidade psicológica os impedem de se adaptar às mudanças nos contextos internos ou externos (Hayes et al., 2012). Dessa forma, recomenda-se que as sessões descritas sirvam como guias do encadeamento lógico a ser seguido em uma intervenção grupal baseada na ACT. Contudo, como característica das terapias contextuais, é de suma importância que o contexto terapêutico seja estruturado a partir do pragmatismo funcional, buscando utilidade na intervenção, para que os indivíduos se aproximem da vida que acreditam valer a pena ser vivida (Hayes et al., 1999). Isso implica em aplicar o protocolo com flexibilidade e criatividade, levando em consideração o contexto em que será desenvolvido e a necessidade do grupo com relação aos processos centrais da ACT.

O período de formação acadêmica é, em geral, um período de transição que pode gerar grande desconforto. Entretanto, é também neste momento sensível que habilidades desenvolvidas podem se con solidar pelo resto da vida (Pistorello, 2013). A partir da perspectiva da ACT, o objetivo é o desenvolvimento de habilidades para lidar com o sofrimento, e não especificamente o esbatimento de sintomas. Nesse sentido, esta intervenção pode servir como uma estratégia útil tanto para o contexto (universidade) como para a problemática (ansiedade). A partir do desenvolvimento de maior flexibilidade psicológica neste ambiente, uma série de dificuldades e problemas típicos do meio acadêmico podem ser contornados. Apesar de a presença da dor ser onipresente, a forma como ela será enfrentada pode ser diferente (Hayes et al., 2012).

Adicionalmente, faz-se necessário ressaltar que os dados aqui apresentados devem ser interpretados com cautela, à luz de suas limitações. A principal limitação refere-se ao tamanho da amostra. Os ensaios clínicos randomizados sobre a aplicabilidade da ACT em grupo para manejo de ansiedade apresentam, em geral, amostras maiores do que a do presente estudo (Coto-Lesmes et al., 2020; Kocovski et al., 2013). Apesar de o número recomendável de participantes em grupos terapêuticos ser de aproximadamente 10 integrantes (Delitti \& Derdyk, 2008; Neufeld, 2017; Pistorello, 2013), a condução concomitante de mais de um grupo, com o mesmo protocolo, pode contornar essa limitação. Não foi realizado cálculo de tamanho amostral, o que, associado ao número reduzido de participantes, aumenta a probabilidade do erro tipo II (incapacidade de encontrar associações existentes) e diminui a capacidade de generalização dos dados. Em segundo lugar, a perda de acompanhamento de três pacientes do grupo controle (mesmo após extensa busca por eles) gera menor poder de comparabilidade. Por fim, não foi feito follow-up dos pacientes, não sendo possível verificar a manutenção dos benefícios alcançados com a intervenção no decorrer do tempo. Dessa forma, os achados desta pesquisa devem ser interpretados no âmbito de resultados preliminares. Sugere-se que estudos futuros com amostras maiores e que acompanhem os sujeitos após a intervenção sejam desenvolvidos para confirmar ou refutar os resultados desta investigação.

\section{Considerações finais}

O estudo consistiu na construção de um protocolo de tratamento grupal semiestruturado para ansiedade e avaliou sua eficácia e efetividade para manejo de ansiedade entre universitários. A ntervenção mostrou-se eficaz para aqueles que a completaram e efetiva para o contexto universitário e suas demandas. De forma geral, este tratamento se mostrou capaz de reduzir a sintomatologia ansiosa em $39,7 \%$ entre os pacientes que completaram o tratamento e em $30,8 \%$ entre todos alocados para o grupo intervenção (incluindo as desistências), enquanto os controles não tiveram redução significativa no mesmo período. Destaca-se que um dos pontos fortes desta intervenção foi a baixa taxa de abandono, possível reflexo da estratégia de busca ativa de possíveis desistentes. A melhora atingida pelos participantes pode ter resultado de um aumento da flexibilidade psicológica. Dessa forma, é plausível que tenha ocorrido redução da esquiva experiencial, contribuindo para uma vida menos julgadora e mais comprometida com aquilo que se mostrou importante para os pacientes ao longo do processo. Portanto, recomenda-se a utilização da intervenção aqui apresentada dentro das instituições de ensino superior como uma alternativa viável para o manejo de sintomatologia ansiosa em níveis clínicos. 


\section{Referências}

Amorim, P. (2000). Mini International Neuropsychiatric Interview (MINI): Validação de entrevista breve para diagnóstico de transtornos mentais. Revista Brasileira de Psiquiatria, 22(3), 106-114. https://doi.org/10.1590/ S1516-44462000000300003

Auerbach, R. P., Alonso, J., Axinn, W. G., Cuijpers, P., Ebert, D. D., Green, J. G., Hwang, I., Liu, H., Mortier, P., Nock, M. K., Pinder-Amaker, S., Sampson, N. A., Aguilar-Gaxiola, S., Al-Hamzawi, A., Andrade, L. H., Benjet, C., Caldas-deAlmeida, J. M., Demyttenaere, K., Florescu, S., ... \& Bruffaerts, R. (2016). Mental disorders among college students in the World Health Organization World Mental Health Surveys. Psychological Medicine, 46(14), 1-16. https:// doi.org/10.1017/S0033291716001665

Bandelow, B., Michaelis, S., \&Wedekind, D. (2017). Treatment of anxiety disorders. Dialogues in Clinical Neuroscience, 19(2), 93-107. http://doi.org/10.31887/DCNS.2017.19.2/bbandelow

Barlow, D., \& Durand, V. M. (2015). Psicopatologia: Uma abordagem integrada (2a ed.). Cengage Learning.

Chagas, M. T., Guilherme, G., \& Moriyama, J. S. (2013). Intervenção clínica em grupo baseada na terapia de aceitação e compromisso: Manejo da ansiedade. Acta Comportamentalia, 21(4), 495-507.

Costa, R. S., \& Soares, M. R. Z. (2015). Terapia de Aceitação e Compromisso: O sofrimento psicológico em um caso clínico. Psicologia: Teoria e Prática, 17(3), 19-27.

Coto-Lesmes, R., Fernández-Rodríguez, C., \& González-Fernández, S. (2020). Acceptance and Commitment Therapy in group format for anxiety and depression. A systematic review. Journal of Affective Disorders, 263, 107120. https://doi.org/10.1016/j.jad.2019.11.154

Daltry, R. M. (2015). A case study: An ACT stress management group in a university counseling center. Journal of College Student Psychotherapy, 29(1), 36-43. https://doi.org/10.1080/87568225.2015.976076

Delitti, M., \& Derdyk, P. (2008). Terapia Analítico Comportamental em Grupo (1a ed.). ESETec Editores Associados.

Demenech, L. M., Oliveira, A. T., Neiva-Silva, L., \& Dumith, S. C. (2021). Prevalence of anxiety, depression and suicidal behaviors among Brazilian undergraduate students: A systematic review and meta-analysis. Journal of Affective Disorders, 282, 147-159. http://doi.org/10.1016/j.jad.2020.12.108

Eifert, G. H., \& Forsyth, J. P. (2014). La terapia de aceptación y compromiso para trastornos de ansiedad (R. Á. Fernández, Trad.; la ed.). Ediciones Mensajero, S.A.

Eilenberg, T., Fink, P., Jensen, J. S., Rief, W., \& Frostholm, L. (2015). Acceptance and commitment group therapy (ACT-G) for health anxiety: A randomized controlled trial. Danish Medical Journal, 63(46), 103-114. https://doi. org/10.1017/S0033291715001579

ElRafihi-Ferreira, R., Morin, C. M., Toscanini, A. C., Lotufo Neto, F., Brasil, I. S., Gallinaro, J. G., Borges, D. S., Conway, S. G., \& Hasan, R. (2020). Acceptance and commitment therapy-based behavioral intervention for insomnia: a pilot randomized controlled trial. Brazilian Journal of Psychiatry, 43(5). https://doi.org/10.1590/1516-4446-2020-0947

England, E. L., Herbert, J. D., Forman, E. M., Rabin, S. J., Juarascio, A., \& Goldstein, S. P. (2012). Acceptance-based exposure therapy for public speaking anxiety. Journal of Contextual Behavioral Science, 1(1), 66-72. https:// doi.org/10.1016/j.jcbs.2012.07.001

Fukahori, L., Silveira, J. M., \& Costa, C. E. (2005). Exibicionismo e procedimentos baseados na Terapia de Aceitação e Compromisso (ACT): Um relato de caso. Revista Brasileira de Terapia Comportamental e Cognitiva, 7(1), 67-76.

Gallagher, R. (2011). National survey of counseling center directors. International Association of Counseling Services.

Germer, C. K., Siegel, R. D., \& Fulton, P. R. (2015). Mindfulness e psicoterapia (2a ed.). Artmed.

Harris, R. (2011). Liberte-se: Evitando as armadilhas da procura da felicidade. Agir.

Hayes-Skelton, S. A., Roemer, L., \& Orsillo, S. M. (2013). A randomized clinical trial comparing an acceptance-based behavior therapy to applied relaxation for generalized anxiety disorder. Journal of Consulting and Clinical Psychology, 81(5), 761-773. http://doi.org/10.1037/a0032871

Hayes, S. C., Barnes-Holmes, D., \& Roche, B. (2002). Relational Frame Theory: A post-skinnerian account of human language and cognition. Kluwer Academic Publishers. 
Hayes, S. C., Luoma, J. B., Bond, F. W., Masuda, A., \& Lillis, J. (2006). Acceptance and Commitment Therapy: Model, processes and outcomes. Behaviour Research and Therapy, 44(1), 1-25. https://doi.org/10.1016/j.brat.2005.06.006

Hayes, S. C., \& Smith, S. (2005). Get out of your mind and into your life: The new Acceptance and Commitment Therapy. New Harbinger Publications, Inc.

Hayes, S. C., Strosahl, K. D., \& Wilson, K. G. (1999). Acceptance and commitment therapy: An experiential approach to behavior change. Guilford Press.

Hayes, S. C., Strosahl, K. D., \& Wilson, K. G. (2012). Acceptance and Commitment Therapy: The process and practice of mindful change (2a ed.). The Guilford Press.

Hayes, S. C., Wilson, K. G., Gifford, E. V., Follette, V. M., \& Strosahl, K. (1996). Experiential avoidance and behavioral disorders: A functional dimensional approach to diagnosis and treatment. Journal of Consulting and Clinical Psychology, 64(6), 1152-1168. https://doi.org/10.1037//0022-006x.64.6.1152

Ibrahim, A. K., Kelly, S. J., Adams, C. E., \& Glazebrook, C. (2013). A systematic review of studies of depression prevalence in university students. Journal of Psychiatric Research, 47(3), 391-400. https://doi.org/10.1016/ j.jpsychires.2012.11.015

Jacobs, M. L., Luci, K., \& Hagemann, L. (2018). Group-based Acceptance and Commitment Therapy (ACT) for older veterans: Findings from a quality improvement project. Clinical Gerontologist, 41(5), 458-467. https://doi.org/ 10.1080/07317115.2017.1391917

Kirkwood, B. R., \& Sterne, J. A. C. (2006). Relaxing model assumptions. In Essential medical statistics (pp. 343-354). Blackwell Publishing Company.

Kocovski, N. L., Fleming, J. E., Hawley, L. L., Huta, V., \& Antony, M. M. (2013). Mindfulness and acceptance-based group therapy versus traditional cognitive behavioral group therapy for social anxiety disorder: A randomized controlled trial. Behaviour Research and Therapy, 51(12), 889-898. http://doi.org/10.1016/j.brat.2013.10.007

Kroenke, K., Spitzer, R. L., \&Williams, J. B. (2001). The PHQ-9:Validity of a brief depression severity measure. Journal of General Internal Medicine, 16(9), 606-613. http://doi.org/10.1046/j.1525-1497.2001.016009606.x

Lucena-Santos, P., Pinto-Gouveia, J., \& Oliveira, M. S. (2015). Terapias comportamentais de terceira geração: Guia para profissionais. Sinopsys.

Machado, D., Soares, M. R. Z., \& Costa, R. S. (2019). Avaliação de uma intervenção em grupo baseada na terapia de aceitação e compromisso para indivíduos diagnosticados com transtorno bipolar. Contextos Clínicos, 12(1), 26-47. https:// doi.org/10.4013/ctc.2019.121.02

Malmir, T., Jafari, H., Ramezanalzadeh, Z., \& Heydari, J. (2017). Determining the effectiveness of Acceptance and Commitment Therapy (ACT) on life expectancy and anxiety among bereaved patients. Materia Socio-Medica, 29(4), 242-246. https://doi.org/10.5455/msm.2017.29.242-246

Moreno, A. L., DeSousa, D. A., Souza, A. M. F. L. P., Manfro, G. G., Salum, G. A., Koller, S. H., Osario, F. L., \& Crippa, J. A. S. (2016). Factor structure, reliability, and item parameters of the Brazilian Portuguese version of the GAD-7 questionnaire. Trends in Psychology, 24(1), 367-375. http://doi.org/10.9788/TP2016.1-25

Neff, K. (2017). Autocompaixão: Pare de se torturar e deixe a insegurança pra trás (B. M. Flores, Trad.). Lúcida Letra.

Neufeld, C. B. (2017). Terapia Cognitivo-Comportamental em grupos: Das evidências à prática (1a ed.). Artmed.

Öst, L.-G. (2014). The efficacy of Acceptance and Commitment Therapy: An updated systematic review and meta-analysis. Behaviour Research and Therapy, 61, 105-121. http://doi.org/10.1016/j.brat.2014.07.018

Pacheco, J. P., Giacomin, H. T., Tam, W. W., Ribeiro, T. B., Arab, C., Bezerra, I. M., \& Pinasco, G. C. (2017). Mental health problems among medical students in Brazil: A systematic review and meta-analysis. Revista Brasileira de Psiquiatria, 39(4), 369-377. https://doi.org/10.1590/1516-4446-2017-2223

Perry, A., Gardener, C., Oliver, J. E., Taş, Ç., \& Özenç, C. (2019). Exploring the cultural flexibility of the ACT model as an effective therapeutic group intervention for Turkish speaking communities in East London. The Cognitive Behaviour Therapist, 12, e2. https://doi.org/10.1017/S1754470X18000041

Pistorello, J. (2013). Mindfulness \& acceptance for counseling college students: Theory and practical applications for intervention, prevention \& outreach. New Harbinger Publications, Inc.

Polk, K. L., Schoendorff, B., Webster, M., \& Olaz, F. O. (2016). The essential guide to the ACT Matrix: A step-by-step approach to using the ACT Matrix Model in clinical practice. New Harbinger Publications, Inc. 
Roemer, L., Orsillo, S. M., \& Salters-Pedneault, K. (2008). Efficacy of an acceptance-based behavior therapy for generalized anxiety disorder: Evaluation in a randomized controlled trial. Journal of Consulting and Clinical Psychology, 76(6), 1083-1088. https://doi.org/10.1037/a0012720

Santos, I. S., Tavares, B. F., Munhoz, T. N., Almeida, L. S. P., Silva, N. T. B., Tams, B. D., Patella, A. M., \& Matijasevich, A. (2013). Sensibilidade e especificidade do Patient Health Questionnaire-9 (PHQ9) entre adultos da população geral. Cadernos de Saúde Pública, 29(8), 1533-1542. https://doi.org/10.1590/S0102-311X2013001200006

Silva, J. L. N., \& de-Farias, A. K. C. R. (2013). Análises funcionais molares associadas à terapia de aceitação e compromisso em um caso de transtorno obsessivo-compulsivo. Revista Brasileira de Terapia Comportamental e Cognitiva, 15(3), 37-56.

Silva, M. T., Caicedo Roa, M., Martins, S. S., Silva, A. T. C. da, \& Galvao, T. F. (2018). Generalized anxiety disorder and associated factors in adults in the Amazon, Brazil: A population-based study. Journal of Affective Disorders, 236, 180-186. http://doi.org/10.1016/j.jad.2018.04.079

Singal, A. G., Higgins, P. D., \& Waljee, A. K. (2014). A primer on effectiveness and efficacy trials. Clinical and Translational Gastroenteroly, 5(1), e45. https://doi.org/10.1038/ctg.2013.13

Sousa, D. D., \& de-Farias, A. K. C. R. (2014). Dor crônica e terapia de aceitação e compromisso: Um caso clínico. Revista Brasileira de Terapia Comportamental e Cognitiva, 16(2), 125-147. https://doi.org/10.31505/rbtcc.v16i2.696

Spitzer, R. L., Kroenke, K., Williams, J. B., \& Lowe, B. (2006). A brief measure for assessing generalized anxiety disorder: The GAD-7. Archives of Internal Medicine, 166(10), 1092-1097. http://doi.org/10.1001/archinte.166.10.1092

Törneke, N. (2010). Learning RFT: An introduction to Relational Frame Theory and its clinical application. New Harbinger Publications, Inc.

Twenge, J. M., Gentile, B., DeWall, C. N., Ma, D., Lacefield, K., \& Schurtz, D. R. (2010). Birth cohort increases in psychopathology among young Americans, 1938-2007: A cross-temporal meta-analysis of the MMPI. Clinical Psychology Review, 30(2), 145-154. https://doi.org/10.1016/j.cpr.2009.10.005

Twohig, M. P., \& Levin, E. L. (2017). Acceptance and commitment therapy as a treatment for anxiety and depression: A review. Psychiatric Clinics of North America, 40(4), 751-769. https:// doi.org/10.1016/j.psc.2017.08.009

Vogel, K., Gordon, P. C., \& Lotufo Neto, F. (2017). Acceptance and commitment therapy for enclosed spaces phobia: A randomized clinical trial. Revista Brasileira de Terapia Comportamental e Cognitiva, 19(3), 9-18. https:// doi.org/10.31505/rbtcc.v19i3.1050

Williams, M., \& Penman, D. (2015). Atenção plena: Mindfulness (1a ed.). Sextante.

Wolgensinger, L. (2015). Cognitive behavioral group therapy for anxiety: Recent developments. Dialogues in Clinical Neuroscience, 17(3), 347-350.

World Health Organization. (2017). Depression and other common mental disorders: Global health estimates.

Zamignani, D. R., \& Banaco, R. A. (2005). Um panorama analítico-comportamental sobre os transtornos de ansiedade. Revista Brasileira de Terapia Comportamental e Cognitiva, 7(1), 77-92. https://doi.org/10.31505/rbtcc.v7il.44

\section{Raimundo Bittencourt de Almeida}

Psicólogo, mestrando no Programa de Pós-Graduação em Psicologia da Universidade Federal do Rio Grande (PPGP/ FURG). Especialização em andamento em Terapia de Aceitação e Compromisso pelo Centro Brasileiro de Ciência Comportamental Contextual (Ceconte). Membro do Centro de Estudos sobre Risco e Saúde da Universidade Federal do Rio Grande (Ceris/Furg), Rio Grande - RS. Brasil.

E-mail: raimundoalmeida.psi@gmail.com

(D) https://orcid.org/0000-0002-6017-3080

\section{Lauro Miranda Demenech}

Doutorando no Programa de Pós-Graduação em Ciências da Saúde da Universidade Federal do Rio Grande (PPGCS/Furg). Vice-coordenador do Centro de Estudos sobre Risco e Saúde (Ceris/Furg), Rio Grande - RS. Brasil

E-mail: auro_demenech@hotmail.com

(D) https://orcid.org/0000-0002-7285-2566 


\section{Paulo Gomes de Sousa-Filho}

Docente do curso de Psicologia e coordenador do Centro de Psicologia Contextual da Universidade Federal do Rio Grande (Cepsico/Furg), Rio Grande - RS. Brasil.

E-mail: paulogomes@furg.br

(D) https://orcid.org/0000-0002-6326-9387

Lucas Neiva-Silva

Docente do curso de Psicologia e do Programa de Pós-Graduação em Psicologia da Universidade Federal do Rio Grande (PPGPsi/Furg). Coordenador do Centro de Estudos sobre Risco e Saúde (Ceris/Furg), Rio Grande - RS. Brasil. E-mail: lucasneivasilva@gmail.com

(iD) https://orcid.org/0000-0002-7526-2238

Endereço para envio de correspondência:

Universidade Federal do Rio Grande. Av. Itália, km 8, Carreiros. CEP: 96201-900. Rio Grande do Sul - RS, Brasil.

Recebido 27/03/2020

Aceito 30/07/2021

Received 03/27/2020

Approved 07/30/2021

Recibido $27 / 03 / 2020$

Aceptado 30/07/2021

Como citar: Almeida, R. B. de, Demenech, L. M., Sousa-Filho, P. G. de, \& Neiva-Silva, L. (2022). ACT em grupo para manejo de ansiedade entre universitários: Ensaio clínico randomizado. Psicologia: Ciência e Profissão, 42,1 -17. https://doi.org/10.1590/1982-3703003235684

How to cite: Almeida, R. B. de, Demenech, L. M., Sousa-Filho, P. G. de, \& Neiva-Silva, L. (2022). Group therapy based on ACT for anxiety management among university students: Randomized Clinical Trial. Psicologia: Ciência e Profissão, 42, 1-17. https://doi.org/10.1590/1982-3703003235684

Cómo citar: Almeida, R. B. de, Demenech, L. M., Sousa-Filho, P. G. de, \& Neiva-Silva, L. (2022). Terapia grupal basada en ACT para el manejo de la ansiedad entre estudiantes universitarios: Ensayo Clínico Aleatorizado. Psicologia: Ciência e Profissão, 42, 1-17. https://doi.org/10.1590/1982-3703003235684 